\title{
Synthesis and Pesticidal Activities of 5-(2-Cyclopropylaminopyrimidin-4-yl)-4-(thiophenyl)thiazole Derivatives
}

\author{
Won-Sik Choi, ${ }^{1}$ Seok-Woo Nam, ${ }^{1}$ Il-Doo Kim, ${ }^{1}$ Seung-Han Kim, ${ }^{1}$ Kun-Ho Park, \\ In-Kyung Bae, ${ }^{3}$ Eun-Sil Park, ${ }^{3}$ Hwang-Ju Jeon, ${ }^{3}$ and Sung-Eun Lee ${ }^{3}$ \\ ${ }^{1}$ Department of Life Science and Biotechnology, Soonchunhyang University, Asan 336-745, Republic of Korea \\ ${ }^{2}$ Department of Chemistry, University of California, Berkeley, CA 94720, USA \\ ${ }^{3}$ School of Applied Biosciences, Kyungpook National University, Daegu 702-701, Republic of Korea
}

Correspondence should be addressed to Sung-Eun Lee; selpest@knu.ac.kr

Received 4 November 2014; Accepted 4 January 2015

Academic Editor: Lutfun Nahar

Copyright $\odot 2015$ Won-Sik Choi et al. This is an open access article distributed under the Creative Commons Attribution License, which permits unrestricted use, distribution, and reproduction in any medium, provided the original work is properly cited.

\begin{abstract}
Pesticidal activities of 4-[5-(2-cyclopropylaminopyrimidin-4-yl)-4-(4-chloro-2-fluoro-phenyl)thiazol-2-yl]-1-methylpiperidine, designated as Comp I, have been determined against a mosquito larva, Culex pipiens pallens, and a phytopathogenic fungus, Phytophthora capsici. Comp I was used as the leading compound in this study. The compounds were synthesized by reacting them with two functional groups, 3-thiophenyl and 2-thiophenyl groups, instead of 4-chloro-2-fluorophenyl group in Comp I. Other functional groups such as 2-aminothiazole, 2-(1-methylpiperazin-4-yl)thiazole, and 2-(piperazin-4-yl)thiazole were also introduced instead of 2-methylpiperidin-4-yl-thiazole of Comp I. Compounds designated as XIII-6 XV-7 were newly synthesized and their structures were confirmed by ${ }^{1} \mathrm{H}$ - and ${ }^{13} \mathrm{C}$-NMR spectroscopy. Mosquito larvicidal activities of all the synthesized compounds against C. pipiens pallens were examined and Comp I among them showed the strongest larvicidal activity as $0.513 \mathrm{mM}_{\text {of }} \mathrm{LC}_{50}$ value. The fungicidal activities of all the synthesized compounds against $P$. capsici were examined using the whole plant method. Among the XIII-6 XV-7 chemicals, 5-(2-cyclopropylaminopyrimidin-4-yl)-4-(thiophen-2-yl)thiazol-2-amine (VIII-6) showed the most potent antifungal activity in vivo. While the $\mathrm{EC}_{50}$ value of the commercial fungicide dimethomorph was $4.26 \mu \mathrm{M}, \mathrm{EC}_{50}$ of VIII-6 was $0.94 \mu \mathrm{M}$. Therefore, thiazole derivatives can be considered as viable candidates for the control of mosquito larvae and plant diseases.
\end{abstract}

\section{Introduction}

The mosquitos have contributed to emerging infectious diseases including malaria, filariasis, dengue, yellow fever, and Japanese encephalitis, leading to death. Among them, vivax malaria reemerges in South Korea and the control strategy for the vectors needs to be changed [1]. The control of mosquitoes using conventional insecticides is one of efficient methods to decrease malaria incidence in South Korea. However, there are many reports that show high insecticide resistance found in the mosquitos in South Korea [2]. Therefore, there is increasing need to develop new insecticides for the control of mosquitoes in public.
Similarly, new antifungal compounds are needed to control phytopathogenic diseases to protect crops from the fungal attack. Among the phytopathogenic diseases, Phytophthora capsici is a soil-borne fungal pathogen that significantly hampers agricultural production of certain species of peppers [3]. Because of its highly destructive effect on infected plants, strategies to detect $P$. capsici and prevent infection by this pathogen have been extensively investigated. This infection can be readily found in pepper and tomato plants in South Korea [4]. To reduce the failure of fungal disease management by the currently used fungicides, new compounds with high efficacy and selectivity against target species are desirable [5]. Several studies on the synthesis of thiazole derivatives have 
already been published [6], reflecting the growing interest in the chemical and biological significance of this class of compounds [7]. 4-[5-(2-Cyclopropylaminopyrimidin-4-yl)-4(4-fluorophenyl)-thiazol-2-yl]-1-methylpiperidine and 4-[5(2-cyclopropylaminopyrimidin-4-yl)-4-(4-chloro-2-fluorophenyl)thiazol-2-yl]-1-methylpiperidine (Comp I) showed potential antifungal activity in vivo $[8,9]$.

The objective of the present study was to develop a new environmentally sound pesticide to control mosquito larvae and the fungus $P$. blight by optimizing novel scaffolds for commercial use. Therefore, based on the structural features of thiazole, compounds that substituted phenyl group of Comp I with 3-thiophenyl or 2-thiophenyl group instead of 4chloro-2-fluorophenyl group were designed and synthesized. The newly synthesized compounds' pesticidal effects were determined on the mosquito larvae and the phytopathogenic fungus.

\section{Materials and Methods}

2.1. Mosquitoes and Pathogens. The stock culture of Culex pipiens pallens was a laboratory strain (KS-CP, Korea National Institute of Health, Osong, Korea) which was susceptible to insecticides and maintained without exposure to further insecticides. P. capsici was kindly supplied by the National Institute of Agricultural Science and Technology (NAAS, Suwon, Korea). This species was subcultured on potato dextrose agar (PDA) (3.9 g) in water $(100 \mathrm{~mL})$ at $15 \mathrm{~atm}$ and $121^{\circ} \mathrm{C}$ for $15 \mathrm{~min}$ and then uniformly sprayed on a Petri dish. $P$. capsici was inoculated onto the medium at intervals of $5 \mathrm{~mm}$ and was then used to test for antifungal activity for $4 \sim 7$ days at $20 \sim 25^{\circ} \mathrm{C}$ and $70 \%$ humidity in a dark room.

2.2. Materials. Piperazine, triethylamine, trifluoroacetic acid, isopentyl nitrite, 1-boc-4-piperidone, ethyl 4-carbamothioyl-piperidine-1-carboxylate, 2-mercapto-4-methylpyrimidine hydrochloride, iodomethane, $\mathrm{O}$-dimethylhydroxyl-amine hydrochloride, thiophene-2-carbonyl chloride, thiophene3 -carbonyl chloride, lithium diisopropylamide (2.0 M), bromine, thiourea, sulfuryl chloride, isonipecotamide, Lawesson's reagent, 3-chloroperoxybenzoic acid, 3-aminobenzyl alcohol, cyclopropylamine, trimethylsilyliodide, formaldehyde solution, and sodium borohydride were obtained from Aldrich Chemical (St. Louis, MO). PDA was supplied from Difco (Sparks, MD). Dimethomorph was supplied from Dongbu Hannong Co. (Daejeon, Korea). ${ }^{1} \mathrm{H}$ - and ${ }^{13} \mathrm{C}$ NMR spectroscopy was carried out on a Bruker 400 NMR spectrometer (Bruker, Ettlingen, Germany). The IR spectra were recorded on a Jasco FT (Fourier Transform)/IR-4100 Fourier transform spectrometer (JASCO, Tokyo, Japan).

2.3. In Vivo Mosquito Larvicidal Activity. A direct-contact mortality bioassay was done on acetone serial dilution of the newly synthesized compounds to third instar larvae of C. pipiens pallens. At least 20 mosquito larvae were separately deposited into a $250 \mathrm{~mL}$ beaker containing each test compound solution with five different concentrations. Chlorpyrifos was served as a positive control and prepared in acetone. All treatments were replicated three times using 20 larvae per replicate. Probit analysis was employed to calculate larvicidal activity of the tested compounds.

2.4. In Vivo Fungicidal Activity. Antifungal activities of the tested compounds were evaluated using the whole plant method in a greenhouse, as previously described $[6,8]$. Briefly, a serial dilution of test compounds was prepared as $40,35,25,20$, and $12.5 \mathrm{mg} / \mathrm{L}$. Fifty $\mathrm{mL}$ of each test sample solution was sprayed into two pots and the treated red pepper plants were left in a greenhouse for 1 day before inoculation with the pathogen. The prepared red pepper plants were then inoculated by spraying conidia $\left(1 \times 10^{4}\right.$ spores $\left./ \mathrm{mL}\right)$ of $P$. capsici on the leaves. Dimethomorph was used as a positive control. All tests were triplicated and the effect of the test compound was evaluated with a control value $(\mathrm{CV})$ calculated using the formula $\mathrm{CV}(\%)=[(A-B) / A] \times 100 . A$ and $B$ represent the disease area on the untreated and treated plants, respectively.

2.5. Statistical Analysis. Analysis of variance was performed with the procedure formulated by SAS version 9.1 (SAS Institute, Cary, NC). If $P>F$ was less than 0.01 , the means were separated with the least significant difference (LSD) test at the $P=0.05$ level.

2.6. Synthesis. Synthesis of compounds designated as VIII-6 and XIII-6 XV-7 is shown in Figure 2. Compounds I, VII, and XII were synthesized by the method previously reported [10]. Compound II was synthesized by the method previously reported [11], and IX and XI were synthesized by the method of [8]. Compound X by the method of [12] and XIII XV compounds [9] were synthesized.

2.7. 5-(2-Cyclopropylaminopyrimidin-4-yl)-4-(thiophenyl)thiazol-2-amine Derivatives (VIII-6 VIII-7). A mixture of 5-(2(methylsulfinyl)pyrimidin-4-yl)-4-(thiophen-2-yl)thiazol2-amine (VII-6) (0.90 g, 3.14 mmole) and 5-(2-(methylsulfinyl)pyrimidin-4-yl)-4-(thiophen-3-yl)thiazol-2-amine (VII-7) $(0.90 \mathrm{~g}, 3.14 \mathrm{mmole})$ in cyclopropylamine $(1.63 \mathrm{~g}$, $28.70 \mathrm{mmole}$ ) was stirred for $2 \mathrm{~h}$ at $100^{\circ} \mathrm{C}$. The mixture was poured into $5 \% \mathrm{HCl}$ solution $(5 \mathrm{~mL})$ and extracted with ethyl acetate $(5 \mathrm{~mL})$. The combined organic phase was dried (magnesium sulfate) and evaporated. The residue was recrystallized from ethyl acetate and hexane to give VIII-6 $(0.80 \mathrm{~g})$ and VIII-7 (0.82 $\mathrm{g})$ as yellow solids.

VIII-6. Yield 81.2\%; ${ }^{1} \mathrm{H}-\mathrm{NMR}\left(400 \mathrm{MHz}, \mathrm{CDCl}_{3}\right) \delta 0.57(\mathrm{~d}$, $\left.2 \mathrm{H},-\mathrm{CCH}_{2} \mathrm{CH}_{2}-\right), 0.77-0.86\left(\mathrm{~d}, 2 \mathrm{H},-\mathrm{CCH}_{2} \mathbf{C H}_{2}-\right)$, 6.71-6.72 $(\mathrm{d}, 1 \mathrm{H},-\mathbf{C H}=\mathrm{CH}-\mathrm{N}), 7.05-7.08(\mathrm{t}, 1 \mathrm{H},-\mathbf{C H}=\mathrm{CH}-\mathrm{S}), 7.38-$ 7.39 (d, 1H, -CH=CH-N), 7.41-7.43 (d, 1H, =CH-S-), 8.11$8.13(\mathrm{~d}, 1 \mathrm{H},=\mathbf{C H}-\mathrm{CH}=\mathrm{CH}-\mathrm{S}) .{ }^{13} \mathrm{C}-\mathrm{NMR}\left(100 \mathrm{MHz}, \mathrm{CDCl}_{3}\right) \delta$ $7.9\left(\mathrm{CH}-\mathrm{CH}_{2}-\mathrm{CH}_{2}\right), 27.0(\mathrm{NH}-\mathrm{CH}), 107.8(\mathrm{C}=\mathbf{C H}-\mathrm{CH}), 122.2$ $(\mathrm{C}=\mathrm{C}-\mathrm{S}, \mathrm{C}), 127.6(\mathrm{C}=\mathbf{C H}-\mathrm{CH}), 129.5(\mathrm{CH}=\mathbf{C H}-\mathrm{CH}), 127.6$ (CH=CH-S), 137.9 (C, S-C=CH) $140.5(\mathrm{C}, \mathrm{N}-\mathrm{C}=\mathrm{C}), 151.2(\mathrm{~N}-$ $\mathrm{CH}=\mathrm{CH}), 159.9(\mathrm{CH}, \mathrm{CH}-\mathrm{C}=\mathrm{N}), 161.1(\mathrm{NH}, \mathrm{N}-\mathrm{C}=\mathrm{N}), 169.2$ $\left(\mathrm{S}, \mathrm{NH}_{2}-\mathrm{C}=\mathrm{N}\right)$. 
VIII-7. Yield 83.2\%; ${ }^{1} \mathrm{H}-\mathrm{NMR}\left(400 \mathrm{MHz}, \mathrm{CDCl}_{3}\right) \delta 0.56(\mathrm{~d}$, $\left.2 \mathrm{H},-\mathrm{CCH}_{2} \mathrm{CH}_{2}-\right), 0.76-0.88$ (d, $\left.2 \mathrm{H},-\mathrm{CCH}_{2} \mathbf{C H}_{2}-\right)$, 6.25$6.27(\mathrm{~d}, 1 \mathrm{H},-\mathrm{CH}=\mathrm{CH}-\mathrm{N}), 7.41-7.84(\mathrm{t}, 1 \mathrm{H},-\mathrm{CH}=\mathrm{CH}-\mathrm{S}), 7.95-$ $8.08(\mathrm{~d}, 1 \mathrm{H},-\mathrm{CH}=\mathbf{C H}-\mathrm{S}), 8.17-8.19(\mathrm{~d}, 1 \mathrm{H},-\mathrm{CH}=\mathbf{C H}-\mathrm{N})$, 8.31-8.52 (d, $1 \mathrm{H},-\mathrm{C}=\mathbf{C H}-\mathrm{S}) .{ }^{13} \mathrm{C}-\mathrm{NMR}\left(100 \mathrm{MHz}, \mathrm{CDCl}_{3}\right)$ $\delta 7.6\left(\mathrm{CH}-\mathrm{CH}_{2}-\mathrm{CH}_{2}\right), 26.0(\mathrm{NH}-\mathrm{CH}), 108.7(\mathrm{C}=\mathbf{C H}-\mathrm{CH})$, $121.1(\mathrm{~S}-\mathbf{C H}=\mathrm{C}), 121.8(\mathrm{C}=\mathrm{C}-\mathrm{S}, \mathrm{C}), 127.8(\mathrm{CH}=\mathbf{C H}-\mathrm{S}), 128.5$ $(\mathrm{CH}=\mathbf{C H}-\mathrm{C}), 142.3(\mathrm{CH}, \mathrm{CH}-\mathrm{C}=\mathrm{C}), 142.2(\mathrm{C}, \mathrm{N}-\mathrm{C}=\mathrm{C}), 158.0$ $(\mathrm{N}-\mathrm{CH}=\mathrm{CH}), 161.9(\mathrm{CH}, \mathrm{CH}-\mathrm{C}=\mathrm{N}), 162.4(\mathrm{NH}, \mathrm{N}-\mathrm{C}=\mathrm{N})$, $166.9\left(\mathrm{~S}, \mathrm{NH}_{2}-\mathrm{C}=\mathrm{N}\right)$.

2.8. Ethyl 5-(2-Cyclopropylaminopyrimidin-4-yl)-4-(thiophenyl)thiazol-2-yl-piperidine-1-carboxylate Derivatives (XIII-6 XIII-7). Application of the VIII method described above afforded XIII-6 (0.43 g) and XIII-7 (0.41 g) as yellow solids.

XIII-6. Yield 68.4\%; ${ }^{1} \mathrm{H}-\mathrm{NMR}\left(400 \mathrm{MHz}, \mathrm{CDCl}_{3}\right) \delta 0.81-0.84$ (d, $\left.4 \mathrm{H},-\mathrm{CH}\left(\mathbf{C H}_{2}\right)_{2}\right), 1.34-1.41\left(\mathrm{t}, 3 \mathrm{H},-\mathrm{CH}_{2} \mathbf{C H}_{3}\right), 1.83-2.18$ (q, $4 \mathrm{H},-\mathrm{CHCH}_{2} \mathrm{CH}_{2}$-), 2.35-3.28 (quin, $1 \mathrm{H},-\mathrm{CHCH}_{2} \mathrm{CH}_{2}$ ), 3.31-3.65 (quin, 4H - $\mathrm{CHCH}_{2} \mathbf{C H}_{2}$ ) , 3.91-4.58 (m, $1 \mathrm{H}$, $\left.-\mathrm{CH}\left(\mathrm{CH}_{2}\right)_{2}\right), 4.68-4.80$ (q, $\left.2 \mathrm{H},-\mathbf{C H}_{2} \mathrm{CH}_{3}\right), 6.65-6.67$ (d, $1 \mathrm{H},-\mathrm{CH}=\mathrm{CH}-\mathrm{N}), 7.41-7.56(\mathrm{t}, 1 \mathrm{H},-\mathrm{CH}=\mathrm{CH}-\mathrm{S}), 8.21-8.23(\mathrm{~d}$, $1 \mathrm{H},-\mathrm{CH}=\mathrm{CH}-\mathrm{N}), 8.45-8.47$ (d, $1 \mathrm{H},=\mathbf{C H}-\mathrm{S}-), 8.54-8.56(\mathrm{~d}$, $1 \mathrm{H},=\mathbf{C H}-\mathrm{CH}=\mathrm{CH}-\mathrm{S}) .{ }^{13} \mathrm{C}-\mathrm{NMR}\left(100 \mathrm{MHz}, \mathrm{CDCl}_{3}\right) \delta 7.4$ $\left(\mathrm{CH}-\mathrm{CH}_{2}-\mathrm{CH}_{2}\right), 13.8\left(\mathrm{CH}_{2}-\mathrm{CH}_{3}\right), 27.1\left(\mathrm{NH}-\mathrm{CH}-\mathrm{CH}_{2}, \mathrm{CH}_{2}\right)$, $29.1\left(\mathrm{CH}-\mathrm{CH}_{2}-\mathrm{CH}_{2}\right), 37.2\left(\mathrm{C}-\mathbf{C H}-\mathrm{CH}_{2}, \mathrm{CH}_{2}\right), 46.1\left(\mathrm{CH}_{2}-\right.$ $\left.\mathrm{CH}_{2}-\mathrm{N}\right), 62.0\left(\mathrm{O}-\mathrm{CH}_{2}-\mathrm{CH}_{3}\right), 107.8(\mathrm{CH}-\mathrm{CH}=\mathrm{C}), 127.6(\mathrm{CH}-$ $\mathbf{C H}=\mathrm{C}), 128.0(\mathrm{CH}-\mathrm{CH}=\mathrm{CH}), 128.6(\mathrm{CH}=\mathrm{C}-\mathrm{S}), 132.9(\mathrm{C}, \mathrm{S}-$ $\mathrm{C}=\mathrm{C}), 139.9(\mathrm{C}, \mathrm{S}-\mathrm{C}=\mathrm{CH}), 146.3(\mathrm{C}, \mathrm{N}-\mathrm{C}=\mathrm{C}), 155.4(\mathrm{~N}, \mathrm{O}-$ $\mathrm{C}=\mathrm{O}), 157.2(\mathrm{~N}=\mathrm{CH}-\mathrm{CH}), 157.7(\mathrm{~N}, \mathrm{C}-\mathrm{C}=\mathrm{CH}), 162.0(\mathrm{NH}, \mathrm{N}-$ $\mathrm{C}=\mathrm{N}), 175.6(\mathrm{~S}, \mathrm{CH}-\mathrm{C}=\mathrm{N})$.

XIII-7. Yield 67.3\%; ${ }^{1} \mathrm{H}-\mathrm{NMR}\left(400 \mathrm{MHz}, \mathrm{CDCl}_{3}\right) \delta 0.85-0.87$ (d, $\left.4 \mathrm{H},-\mathrm{CH}\left(\mathbf{C H}_{2}\right)_{2}\right), 1.35-1.48$ (t, $\left.3 \mathrm{H},-\mathrm{CH}_{2} \mathbf{C H}_{3}\right), 1.88-2.14$ (q, $4 \mathrm{H},-\mathrm{CHCH}_{2} \mathrm{CH}_{2}$-), 2.37-3.21 (quin, $1 \mathrm{H},-\mathrm{CHCH}_{2} \mathrm{CH}_{2}$ ), 3.43-3.61 (quin, $4 \mathrm{H}-\mathrm{CHCH}_{2} \mathbf{C H}_{2}$ ), $3.96-4.51(\mathrm{~m}, 1 \mathrm{H}$, $\left.-\mathrm{CH}\left(\mathrm{CH}_{2}\right)_{2}\right), 4.61-4.86\left(\mathrm{q}, 2 \mathrm{H},-\mathrm{CH}_{2} \mathrm{CH}_{3}\right), 6.63-6.65(\mathrm{~d}, 1 \mathrm{H}$, $-\mathrm{CH}=\mathrm{CH}-\mathrm{N}), 7.41-7.56(\mathrm{t}, 1 \mathrm{H},-\mathrm{CH}=\mathrm{CH}-\mathrm{S}), 7.81-7.85(\mathrm{~d}, 1 \mathrm{H}$, $-\mathrm{CH}=\mathrm{CH}-\mathrm{S}-)$, 8.25-8.27 (d, $1 \mathrm{H},-\mathbf{C H}=\mathrm{CH}-\mathrm{N}), 8.41-8.43$ (d, $1 \mathrm{H},-\mathrm{C}=\mathrm{CH}-\mathrm{S}) .{ }^{13} \mathrm{C}-\mathrm{NMR}\left(100 \mathrm{MHz}, \mathrm{CDCl}_{3}\right) \delta 7.4(\mathrm{CH}-$ $\left.\mathbf{C H}_{2}-\mathrm{CH}_{2}\right), 14.1\left(\mathrm{CH}_{2}-\mathbf{C H}_{3}\right), 27.5\left(\mathrm{NH}-\mathrm{CH}-\mathrm{CH}_{2}, \mathrm{CH}_{2}\right), 29.1$ $\left(\mathrm{CH}-\mathrm{CH}_{2}-\mathrm{CH}_{2}\right), 37.1\left(\mathrm{C}-\mathbf{C H}-\mathrm{CH}_{2}, \mathrm{CH}_{2}\right), 46.5\left(\mathrm{CH}_{2}-\mathbf{C H}_{2}-\right.$ $\mathrm{N}), 62.5\left(\mathrm{O}-\mathrm{CH}_{2}-\mathrm{CH}_{3}\right), 107.5(\mathrm{CH}-\mathrm{CH}=\mathrm{C}), 121.6(\mathrm{~S}-\mathrm{CH}=\mathrm{C})$, $128.2(\mathrm{CH}=\mathbf{C H}-\mathrm{S}), 128.3(\mathrm{CH}=\mathbf{C H}-\mathrm{C}), 132.5(\mathrm{C}, \mathrm{S}-\mathrm{C}=\mathrm{C}), 142.5$ (C, C-C=CH), $146.6(\mathrm{C}, \mathrm{N}-\mathrm{C}=\mathrm{C}), 155.1(\mathrm{~N}, \mathrm{O}-\mathrm{C}=\mathrm{O}), 157.9$ $(\mathrm{N}=\mathrm{CH}-\mathrm{CH}), 157.7(\mathrm{~N}, \mathrm{C}-\mathrm{C}=\mathrm{CH}), 162.0(\mathrm{NH}, \mathrm{N}-\mathrm{C}=\mathrm{N}), 175.8$ $(\mathrm{S}, \mathrm{CH}-\mathrm{C}=\mathrm{N})$.

2.9. 5-(2-Cyclopropylaminopyrimidin-4-yl)-4-(thiophenyl)thiazol-2-yl-piperidine Derivatives (XIV-6 XIV-7). Trimethylsilyliodide $(1.27 \mathrm{~g}, 6.36 \mathrm{mM})$ was added to a solution of XIII$6(0.85 \mathrm{~g}, 1.88 \mathrm{mmole})$ and XIII-7 (0.85 g, $1.88 \mathrm{mmole})$, in chloroform $10 \mathrm{~mL}$. After the addition was completed, the resulting mixture was stirred at $60^{\circ} \mathrm{C}$ for $4 \mathrm{~h}$. Subsequently, $10 \mathrm{~mL}$ of $6 \mathrm{M} \mathrm{HCl}$ in isopropanol and $10 \mathrm{~mL}$ of $2 \mathrm{M} \mathrm{NaOH}$ solution were added to the mixture. The organic phase was then separated, dried (magnesium sulfate), and evaporated to yield a residue. The residue was recrystallized from methylene chloride and ether to give XIV-6 (0.50 g) and XIV-7 (0.44 g) as yellow solids.

XIV-6. Yield 52.5\%; ${ }^{1} \mathrm{H}-\mathrm{NMR}\left(400 \mathrm{MHz}, \mathrm{CDCl}_{3}\right) \delta 0.85-$ $0.87\left(\mathrm{~d}, 4 \mathrm{H},-\mathrm{CH}\left(\mathbf{C H}_{2}\right)_{2}\right), 1.81-2.12\left(\mathrm{q}, 4 \mathrm{H},-\mathrm{CHCH}_{2} \mathrm{CH}_{2}-\right.$ ), 2.33-3.24 (quin, $1 \mathrm{H},-\mathbf{C H C H}_{2} \mathrm{CH}_{2}-$ ), 3.36-3.63 (quin, $4 \mathrm{H}$, - $\left.\mathrm{CHCH}_{2} \mathbf{C H}_{2}-\right)$, 3.95-4.54 (m, $\left.1 \mathrm{H},-\mathrm{CH}\left(\mathrm{CH}_{2}\right)_{2}\right), 6.62-6.65$ (d, $1 \mathrm{H},-\mathbf{C H}=\mathrm{CH}-\mathrm{N}), 7.46-7.57$ ( $\mathrm{t}, 1 \mathrm{H},-\mathbf{C H}=\mathrm{CH}-\mathrm{S}), 8.25-8.27$ $(\mathrm{d}, 1 \mathrm{H},-\mathrm{CH}=\mathbf{C H}-\mathrm{N}), 8.43-8.45(\mathrm{~d}, 1 \mathrm{H},=\mathbf{C H}-\mathrm{S}-), 8.51-8.53$ $(\mathrm{d}, \mathrm{H},=\mathrm{CH}-\mathrm{CH}=\mathrm{CH}-\mathrm{S}) .{ }^{13} \mathrm{C}-\mathrm{NMR}\left(100 \mathrm{MHz}, \mathrm{CDCl}_{3}\right) \delta 7.4$ $\left(\mathrm{CH}-\mathrm{CH}_{2}-\mathrm{CH}_{2}\right), 28.0\left(\mathrm{NH}-\mathrm{CH}-\mathrm{CH}_{2}, \mathrm{CH}_{2}\right), 32.4\left(\mathrm{CH}-\mathrm{CH}_{2}-\right.$ $\left.\mathrm{CH}_{2}\right), 39.5\left(\mathrm{C}-\mathrm{CH}-\mathrm{CH}_{2}, \mathrm{CH}_{2}\right), 41.8\left(\mathrm{CH}_{2}-\mathrm{CH}_{2}-\mathrm{NH}\right), 107.8$ $(\mathrm{CH}-\mathrm{CH}=\mathrm{C}), 127.6(\mathrm{CH}-\mathrm{CH}=\mathrm{C}), 128.0(\mathrm{CH}-\mathrm{CH}=\mathrm{CH}), 129.6$ $(\mathrm{CH}=\mathrm{C}-\mathrm{S}), 133.2(\mathrm{C}, \mathrm{S}-\mathrm{C}=\mathrm{C}), 139.8(\mathrm{C}, \mathrm{S}-\mathrm{C}=\mathrm{CH}), 146.3(\mathrm{C}$, $\mathrm{N}-\mathrm{C}=\mathrm{C}), 157.2(\mathrm{~N}=\mathrm{CH}-\mathrm{CH}), 158.8(\mathrm{~N}, \mathrm{C}-\mathrm{C}=\mathrm{CH}), 162.0(\mathrm{NH}$, $\mathrm{N}-\mathrm{C}=\mathrm{N}), 175.5(\mathrm{~S}, \mathrm{CH}-\mathrm{C}=\mathrm{N})$.

XIV-7. Yield 52.2\%; ${ }^{1} \mathrm{H}-\mathrm{NMR}\left(400 \mathrm{MHz}, \mathrm{CDCl}_{3}\right) \delta 0.88-$ 0.90 (d, $\left.4 \mathrm{H},-\mathrm{CH}\left(\mathbf{C H}_{2}\right)_{2}\right), 1.84-2.15$ (q, $4 \mathrm{H},-\mathrm{CHCH}_{2} \mathrm{CH}_{2}$ ) , 2.32-3.24 (quin, $1 \mathrm{H},-\mathrm{CHCH}_{2} \mathrm{CH}_{2}-$ ), 3.41-3.63 (quin, $4 \mathrm{H}$, $\left.-\mathrm{CHCH}_{2} \mathbf{C H}_{2}-\right), 3.95-4.55\left(\mathrm{~m}, 1 \mathrm{H},-\mathbf{C H}\left(\mathrm{CH}_{2}\right)_{2}\right), 6.62-6.64(\mathrm{~d}$, $1 \mathrm{H},-\mathrm{CH}=\mathrm{CH}-\mathrm{N}), 7.43-7.52(\mathrm{t}, 1 \mathrm{H},-\mathbf{C H}=\mathrm{CH}-\mathrm{S}), 7.86-7.88(\mathrm{~d}$, $1 \mathrm{H},-\mathrm{CH}=\mathbf{C H}-\mathrm{S}-), 8.22-8.24(\mathrm{~d}, 1 \mathrm{H},-\mathrm{CH}=\mathbf{C H}-\mathrm{N}), 8.45-8.47$ $(\mathrm{d}, 1 \mathrm{H},-\mathrm{C}=\mathrm{CH}-\mathrm{S}) .{ }^{13} \mathrm{C}-\mathrm{NMR}\left(10 \mathrm{MHz}, \mathrm{CDCl}_{3}\right) \delta 7.4(\mathrm{CH}-$ $\left.\mathrm{CH}_{2}-\mathrm{CH}_{2}\right), 28.0\left(\mathrm{NH}-\mathrm{CH}-\mathrm{CH}_{2}, \mathrm{CH}_{2}\right), 32.3\left(\mathrm{CH}-\mathrm{CH}_{2}-\mathrm{CH}_{2}\right)$, $39.6\left(\mathrm{C}-\mathbf{C H}-\mathrm{CH}_{2}, \mathrm{CH}_{2}\right), 41.0\left(\mathrm{CH}_{2}-\mathbf{C H}_{2}-\mathrm{NH}\right), 107.9(\mathrm{CH}-$ $\mathbf{C H}=\mathrm{C}), 121.7(\mathrm{~S}-\mathbf{C H}=\mathrm{C}), 129.0(\mathrm{CH}=\mathbf{C H}-\mathrm{C}), 129.5(\mathrm{CH}=\mathbf{C H}-$ S), 132.9 (C, S-C=C), $142.3(\mathrm{CH}-\mathrm{C}=\mathrm{CH}), 147.3(\mathrm{C}, \mathrm{N}-\mathrm{C}=\mathrm{C})$, $157.7(\mathrm{~N}, \mathrm{C}-\mathrm{C}=\mathrm{CH}), 158.2(\mathrm{~N}=\mathrm{CH}-\mathrm{CH}), 161.9(\mathrm{NH}, \mathrm{N}-\mathrm{C}=\mathrm{N})$, $176.0(\mathrm{~S}, \mathrm{CH}-\mathrm{C}=\mathrm{N})$.

2.10. 5-(2-Cyclopropylaminopyrimidin-4-yl)-4-(thiophenyl)thiazol-2-yl-1-methylpiperidine Derivatives $(X V-6 \sim X V-7)$. Formaldehyde $(0.13 \mathrm{~g}, 5.02 \mathrm{mM})$ and sodium borohydride $(0.16 \mathrm{~g}, 5.02 \mathrm{mM})$ were added to a solution of XIV-6 $(0.82 \mathrm{~g}$, $2.17 \mathrm{mmole})$ and XIV-7 $(0.82 \mathrm{~g}, 2.17 \mathrm{mmole})$, in methanol $(10 \mathrm{~mL})$. After accumulation, the resulting mixture was stirred at room temperature for $30 \mathrm{~min}$, followed by the addition of $10 \mathrm{~mL}$ water and $10 \mathrm{~mL}$ ethyl acetate. The organic phase was then separated, dried (magnesium sulfate), and evaporated to yield residue. The residue was recrystallized from methylene chloride and ether to give XV-6 (0.31 g) and $\mathrm{XV}-7(0.32 \mathrm{~g})$ as yellow solids.

$X V$-6. Yield $36.5 \%$; ${ }^{1} \mathrm{H}-\mathrm{NMR}\left(400 \mathrm{MHz}, \mathrm{CDCl}_{3}\right) \delta 0.85-$ 0.87 (d, $\left.4 \mathrm{H},-\mathrm{CH}\left(\mathbf{C H}_{2}\right)_{2}\right), 1.81-2.12$ (q, $4 \mathrm{H},-\mathrm{CHCH}_{2} \mathrm{CH}_{2}-$ ), 2.20 (s, 3H, $-\mathrm{NCH}_{3}$ ), 2.33-3.24 (quin, $1 \mathrm{H},-\mathrm{CHCH}_{2} \mathrm{CH}_{2}$ ), 3.36-3.63 (quin, $4 \mathrm{H},-\mathrm{CHCH}_{2} \mathbf{C H}_{2}$ ) $)$ 3.95-4.54 (m, $1 \mathrm{H}$, $\left.-\mathrm{CH}\left(\mathrm{CH}_{2}\right)_{2}\right), 6.62-6.65(\mathrm{~d}, 1 \mathrm{H},-\mathrm{CH}=\mathrm{CH}-\mathrm{N}), 7.46-7.57(\mathrm{t}, 1 \mathrm{H}$, $-\mathbf{C H}=\mathrm{CH}-\mathrm{S}), 8.25-8.27(\mathrm{~d}, 1 \mathrm{H},-\mathrm{CH}=\mathbf{C H}-\mathrm{N}), 8.43-8.45(\mathrm{~d}$, $1 \mathrm{H},=\mathbf{C H}-\mathrm{S}-), 8.51-8.53(\mathrm{~d}, 1 \mathrm{H},=\mathbf{C H}-\mathrm{CH}=\mathrm{CH}-\mathrm{S}) .{ }^{13} \mathrm{C}-\mathrm{NMR}$ $\left(100 \mathrm{MHz}, \mathrm{CDCl}_{3}\right) \delta 7.5\left(\mathrm{CH}-\mathrm{CH}_{2}-\mathrm{CH}_{2}\right), 28.1\left(\mathrm{NH}-\mathrm{CH}-\mathrm{CH}_{2}\right.$, $\left.\mathrm{CH}_{2}\right), 29.9\left(\mathrm{CH}-\mathrm{CH}_{2}-\mathrm{CH}_{2}\right), 38.5\left(\mathrm{C}-\mathbf{C H}-\mathrm{CH}_{2}, \mathrm{CH}_{2}\right), 46.9$ $\left(\mathrm{N}-\mathrm{CH}_{3}\right), 58.8\left(\mathrm{CH}_{2}-\mathrm{CH}_{2}-\mathrm{N}\right), 105.9(\mathrm{CH}-\mathrm{CH}=\mathrm{C}), 128.4(\mathrm{CH}-$ $\mathbf{C H}=\mathrm{C}), 128.7(\mathrm{CH}-\mathrm{CH}=\mathrm{CH}), 129.6(\mathrm{CH}=\mathrm{C}-\mathrm{S}), 133.2(\mathrm{C}, \mathrm{S}-$ $\mathrm{C}=\mathrm{C}), 139.6(\mathrm{C}, \mathrm{S}-\mathrm{C}=\mathrm{CH}), 146.3(\mathrm{C}, \mathrm{N}-\mathrm{C}=\mathrm{C}), 157.1(\mathrm{~N}=\mathbf{C H}-$ $\mathrm{CH}), 158.7(\mathrm{~N}, \mathrm{C}-\mathrm{C}=\mathrm{CH}), 161.8(\mathrm{NH}, \mathrm{N}-\mathrm{C}=\mathrm{N}), 176.6(\mathrm{~S}, \mathrm{CH}-$ $\mathrm{C}=\mathrm{N})$. 
TABLE 1: $\mathrm{EC}_{50}$ and $\mathrm{LC}_{50}$ values of pesticidal activities of VIII-6, VIII-7, XIV-6, XV-6, and XV-7 against P. capsici and CX.p. pallens.

\begin{tabular}{|c|c|c|}
\hline Compounds & $\mathrm{EC}_{50}\left(\mathrm{EC}_{90}\right)$ values $(\mathrm{mM})^{\mathrm{a}}$ & $\begin{array}{c}\mathrm{LC}_{50}(\mathrm{mM}) \\
(\mathrm{CL}, \text { confident limits })\end{array}$ \\
\hline VIII-6 & $0.94 \pm 0.01(4.21 \pm 0.05)$ & - \\
\hline VIII-7 & $3.19 \pm 0.02(9.78 \pm 0.03)$ & - \\
\hline XIV-6 & $3.49 \pm 0.07(12.17 \pm 0.04)$ & - \\
\hline $\mathrm{XV}-6$ & $0.96 \pm 0.03(5.64 \pm 0.01)$ & - \\
\hline $\mathrm{XV}-7$ & $4.30 \pm 0.05(15.31 \pm 0.02)$ & - \\
\hline Comp I & $0.98 \pm 0.21(5.85 \pm 0.05)$ & $0.513(0.290-0.704)$ \\
\hline Dimethomorph & $4.26 \pm 0.02(14.72 \pm 0.05)$ & \\
\hline Chlorpyrifos & & $0.0035(0.0024-0.0047)$ \\
\hline
\end{tabular}

$X V$-7. Yield 37.2\%; ${ }^{1} \mathrm{H}-\mathrm{NMR}\left(400 \mathrm{MHz}, \mathrm{CDCl}_{3}\right) \delta 0.88-$ $0.90\left(\mathrm{~d}, 4 \mathrm{H},-\mathrm{CH}\left(\mathbf{C H}_{2}\right)_{2}\right), 1.84-2.15\left(\mathrm{q}, 4 \mathrm{H},-\mathrm{CHCH}_{2} \mathrm{CH}_{2}-\right)$, $2.26\left(\mathrm{~s}, 3 \mathrm{H},-\mathrm{NCH}_{3}\right), 2.32-3.24$ (quin, $1 \mathrm{H},-\mathrm{CHCH}_{2} \mathrm{CH}_{2}-$ ), 3.41-3.63 (quin, $4 \mathrm{H},-\mathrm{CHCH}_{2} \mathbf{C H}_{2^{-}}$), $3.95-4.55(\mathrm{~m}, 1 \mathrm{H}$, $\left.-\mathrm{CH}\left(\mathrm{CH}_{2}\right)_{2}\right), 6.62-6.64(\mathrm{~d}, 1 \mathrm{H},-\mathrm{CH}=\mathrm{CH}-\mathrm{N}), 7.43-7.52(\mathrm{t}, 1 \mathrm{H}$, $-\mathbf{C H}=\mathrm{CH}-\mathrm{S}), 7.86-7.88(\mathrm{~d}, 1 \mathrm{H},-\mathrm{CH}=\mathbf{C H}-\mathrm{S}-), 8.22-8.24(\mathrm{~d}$, $1 \mathrm{H},-\mathrm{CH}=\mathrm{CH}-\mathrm{N}), 8.45-8.47(\mathrm{~d}, 1 \mathrm{H},-\mathrm{C}=\mathbf{C H}-\mathrm{S}) .{ }^{13} \mathrm{C}-\mathrm{NMR}$ $\left(100 \mathrm{MHz}, \mathrm{CDCl}_{3}\right) \delta 7.5\left(\mathrm{CH}-\mathrm{CH}_{2}-\mathrm{CH}_{2}\right), 28.1\left(\mathrm{NH}-\mathrm{CH}-\mathrm{CH}_{2}\right.$, $\left.\mathrm{CH}_{2}\right), 29.8\left(\mathrm{CH}-\mathrm{CH}_{2}-\mathrm{CH}_{2}\right), 38.5\left(\mathrm{C}-\mathbf{C H}-\mathrm{CH}_{2}, \mathrm{CH}_{2}\right), 46.9$ $\left(\mathrm{N}-\mathrm{CH}_{3}\right), 58.9\left(\mathrm{CH}_{2}-\mathrm{CH}_{2}-\mathrm{NH}\right), 107.7(\mathrm{CH}-\mathrm{CH}=\mathrm{C}), 121.5$ (S$\mathbf{C H}=\mathrm{C}), 128.9(\mathrm{CH}=\mathbf{C H}-\mathrm{S}), 129.8(\mathrm{CH}=\mathbf{C H}-\mathrm{C}), 132.9$ (C, S$\mathrm{C}=\mathrm{C}), 142.3(\mathrm{CH}-\mathrm{C}=\mathrm{CH}), 146.3(\mathrm{C}, \mathrm{N}-\mathrm{C}=\mathrm{C}), 157.7(\mathrm{~N}, \mathrm{C}-$ $\mathrm{C}=\mathrm{CH}), 158.7(\mathrm{~N}=\mathrm{CH}-\mathrm{CH}), 161.8(\mathrm{NH}, \mathrm{N}-\mathrm{C}=\mathrm{N}), 176.6(\mathrm{~S}$, $\mathrm{CH}-\mathrm{C}=\mathrm{N}$ ).

\section{Results}

To obtain new synthesized compounds (VIII-6 VIII-7 and XIII-6 XV-7) from Comp I (Figure 1), 4-chloro-2fluorophenyl group of Comp I was replaced with thiophen3 -yl and thiophen-2-yl groups. In addition, 4-aminothiazole, piperazin-4-yl-carboxylate, 2-(1-methylpiperazin-4-yl)thiazole, and 2-(piperazin-4-yl)thiazole were introduced as the leads instead of the 2-methyl piperidin-4-yl-thiazole group of Comp I. The synthesized compounds with this scheme are shown in Figure 2. Structure of compounds (XIII-6 XV-7) were confirmed by ${ }^{1} \mathrm{H}$ - and ${ }^{13} \mathrm{C}$-NMR spectroscopy.

Larvicidal activities of the synthesized compounds were determined (Table 1) and Comp I showed the strongest activity in the tested compounds as the $\mathrm{LC}_{50}$ value of $0.513 \mathrm{mM}$. However, other synthesized compounds did not show any larvicidal activity against the $C$. pipiens pallens.

Fungicidal activities of the synthesized compounds were determined against $P$. capsici. Comp I exhibited a strong inhibitory action with $\mathrm{EC}_{50}$ value of $0.98 \mathrm{mM}$ on the growth of $P$. capsici, whereas that of the commercial fungicide dimethomorph was $4.26 \mathrm{mM}$ (Table 1). VIII-6 and XV-6 had potent fungicidal activities among the tested compounds with $\mathrm{EC}_{50}$ values of 0.94 and $0.96 \mathrm{mM}$, respectively.

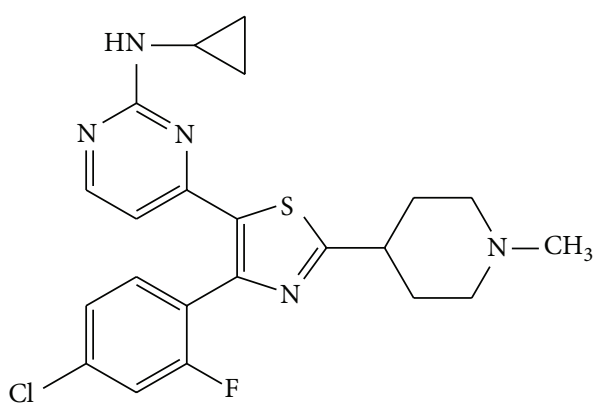

FIGURE 1: Structure of 4-[5-(2-cyclopropylaminopyrimidin-4-yl)-4(4-chloro-2-fluoro-phenyl)thiazol-2-yl]-1-methylpiperidine (Comp I).

\section{Discussion}

The conventional insecticides have been used to control mosquito-borne diseases by decreasing number of mosquito adults as well as larvae. However, many studies have reported that the resistance in mosquitoes to insecticides is common and increasing over the world [2]. The occurrence of resistance in insect pests to conventional insecticides has evoked to study new compounds with novel mode of action. Recently, several reports have found some natural products having mosquito larvicidal activity and antifungal activity [13]. The authors reported eleven compounds isolated from the heartwood of Mansonia gagei showed their larvicidal activities against Aedes aegypti, as well as their antifungal activities against Cladosporium cucumerinum and Candida albicans [13]. It is interesting that one compound may possess biological activities to control both fungi and insects. Mansonone $\mathrm{C}$ was the most potent compound with antifungal and larvicidal properties in the plant $M$. gagei. On the other hand, new synthesized compounds can contribute to control of mosquito larvae as well as natural products [14]. They synthesized 20 isoxaole derivatives and found potent larvicidal activities towards A. aegypti larvae [14]. 


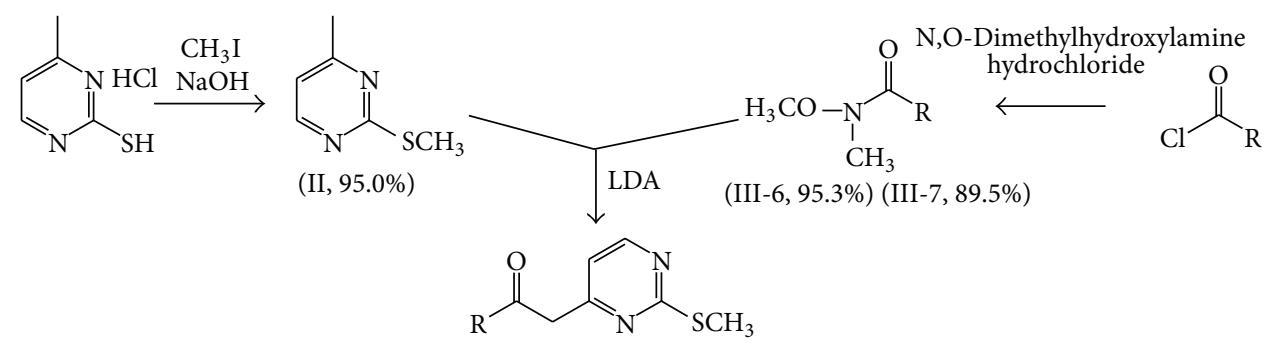

(IV-6, 56.3\%) (IV-7, 50.4\%)<smiles>[R]c1nc(N)sc1-c1ccnc(SC)n1</smiles>

(VI-6, 82.7\%) (VI-7, 81.2\%)<smiles>[R]c1nc(N)sc1-c1ccnc(S(C)=O)n1</smiles>

(VII-6) (VII-7) not isolated<smiles>[R]c1nc(N)sc1-c1ccnc(NC2CC2CC)n1</smiles>

(VIII-6, 81.2\%) (VIII-7, 83.2\%)

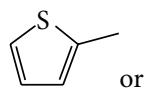

(6)

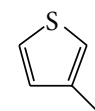

(7)

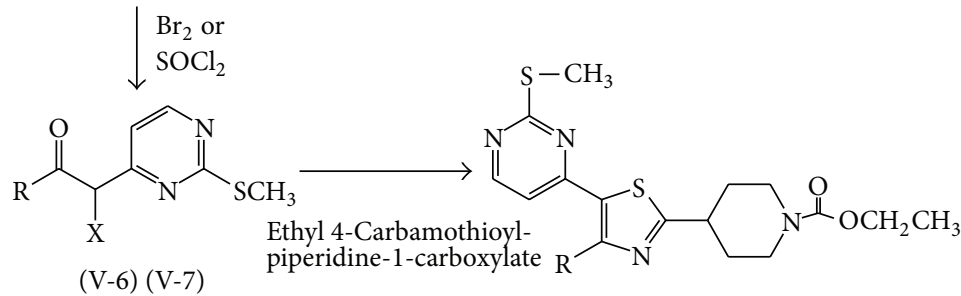

(XI-6, 68.4\%) (XI-7, 67.2\%)<smiles>[R]c1nc(C2CCN(C(=O)OCC)CC2)sc1-c1ccnc(S(C)=O)n1</smiles>

(XII-6) (XII-7) not isolated<smiles>[R]c1nc(C2CCN(C(=O)OCC)CC2)sc1-c1ccnc(NC2CC2)n1</smiles>

(XIII-6, 68.4\%) (XIII-7, 67.3\%)

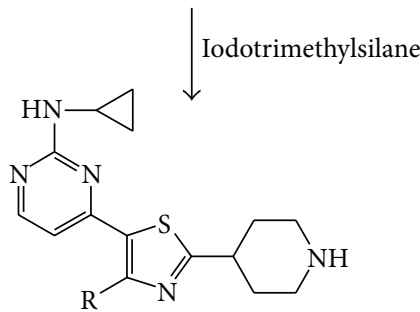

(XIV-6, 52.5\%) (XIV-7, 52.2\%)

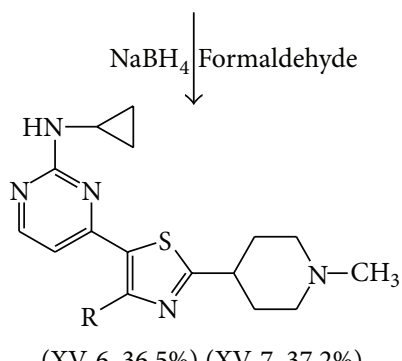

(XV-6, 36.5\%) (XV-7, 37.2\%)

FIGURE 2: Synthesis of 5-(2-cyclopropylaminopyrimidin-4-yl)-4-(thiophenyl)thiazole-2-yl-2-amine (VIII) and 4-[5-(2-cyclopropylaminopyrimidin-4-yl)-4-(thiophenyl)thiazol-2-yl]piperidine derivatives (VIX-6 XV-7). 
Our previous studies were focused on developing new antifungal compounds to selectively control $P$. capsici $[6,8,9]$. Therefore, 4-[5-(2-cyclopropylaminopyrimidin-4-yl)-4-(4chloro-2-fluoro-phenyl)thiazol-2-yl]-1-methylpiperidine (Comp I) is found to show potent antifungal activity towards P. capsici $[6,8,9]$. With these schemes, Comp I appeared to be suitable for use as a leading compound for further synthesis to control $P$. capsici as well as mosquito larvae. A series of new compounds that possessed the thiophenyl group (VIII-6 VIII-7, XIII-6 XV-7) were successfully synthesized in our laboratory and their pesticidal activities towards P. capsici as well as mosquito larvae of $C$. pipiens pallens were determined. The synthesized compounds showed their potent fungicidal effects on $P$. capsici as shown in Table 1. However, only Comp I showed a strong larvicidal activity towards mosquito larvae of C. pipiens pallens (Table 1).

With regard to structure-activity relationship of the newly synthesized compound VIII-6, the functional group on the thiazole ring of compounds such as 2-thiophenyl group may play an important role in the fungicidal activity against $P$. capsici. A dramatic fungicidal effect was found in the compounds that possessed the 2-thiophenyl group on the thiazole ring designated as VIII-6. On the other hand, amine of piperidine groups containing 2-amino group among the newly synthesized compounds also showed fungicidal activity on P. capsici, and VIII-6 containing 2-aminothiazole group increased fungicidal activities with $\mathrm{EC}_{50}$ and $\mathrm{EC}_{90}$ values of 0.94 and $4.21 \mathrm{mM}$, respectively. Therefore, these compounds showed a gradual increase in fungicidal activity in the following order: XV-7, XIV-6, VIII-7, I, XV-6, and VIII6 (Table 1). These data may be extremely useful for developing new fungicides from compounds that include an aryl halide.

Taken together, all results confirmed our original proposal: thiazole compounds mixed with aryl halide groups on the thiazole ring could increase the fungicidal activity. However, these thiazole compounds did not possess any useful larvicidal activity.

\section{Conflict of Interests}

The authors declare that there is no conflict of interests regarding the publication of this paper.

\section{Acknowledgment}

This work was supported by the Soonchunhyang University Research Fund (20120663).

\section{References}

[1] E.-T. Han, D.-H. Lee, K.-D. Park et al., "Reemerging vivax malaria: changing patterns of annual incidence and control programs in the Republic of Korea," The Korean Journal of Parasitology, vol. 44, no. 4, pp. 285-294, 2006.

[2] K.-S. Chang, D.-H. Yoo, E.-H. Shin, W.-G. Lee, J. Y. Roh, and M. Y. Park, "Susceptibility and resistance of field populations of Anopheles sinensis (Diptera: Culicidae) collected from Paju to 13 insecticides," Osong Public Health and Research Perspectives, vol. 4, no. 2, pp. 76-80, 2013.
[3] C. L. Biles, D. L. Lindsey, and C. M. Liddell, "Control of Phytophthora root rot of chile peppers by irrigation practices and fungicides," Crop Protection, vol. 11, no. 3, pp. 225-228, 1992.

[4] M. Rajkumar, W. H. Lee, and K. J. Lee, "Screening of bacterial antagonists for biological control of Phytophthora blight of pepper," Journal of Basic Microbiology, vol. 45, no. 1, pp. 55-63, 2005.

[5] J.-X. Huang, Y.-M. Jia, X.-M. Liang et al., "Synthesis and fungicidal activity of macrolactams and macrolactones with an oxime ether side chain," Journal of Agricultural and Food Chemistry, vol. 55, no. 26, pp. 10857-10863, 2007.

[6] W.-S. Choi, S.-W. Nam, E.-K. Ahn et al., "Synthesis and fungicidal activity of N-[4-(4-fluoro)phenyl-2-piperidin-4-ylthiazol-5-yl]pyrimidin-2-yl-N-phenylamines on Phytophthora capsici," Journal of Applied Biological Chemistry, vol. 53, no. 2, pp. 206-214, 2010.

[7] X. Xu, X. Qian, Z. Li, G. Song, and W. Chen, "Synthesis and fungicidal activity of fluorine-containing phenyliminothiazolidines derivatives," Journal of Fluorine Chemistry, vol. 125, no. 7, pp. 1159-1162, 2004.

[8] S.-W. Nam, I.-Y. Choi, and W.-S. Choi, "Synthesis and antifungal activity of 5-[2-(alkylamino)pyrimidin-4-yl]-4-phenylthiazol2-cycloalkylamine derivatives on Phytophthora capsici," Journal of the Korean Society for Applied Biological Chemistry, vol. 54, no. 3, pp. 395-402, 2011.

[9] S.-W. Nam, G.-R. Lee, T.-J. Kim, B.-J. Chung, and W.-S. Choi, "Synthesis and antifungal activities of 4-[5-(2-cyclopropylaminopyrimidin-2-yl)-4-arylthiazol-5-yl] piperazine derivatives on Phytophthora capsici," The Korean Journal of Pesticide Science, vol. 53, pp. 206-214, 2012.

[10] L. Revesz, E. Blum, F. E. di Padova et al., "Novel p38 inhibitors with potent oral efficacy in several models of rheumatoid arthritis," Bioorganic \& Medicinal Chemistry Letters, vol. 14, no. 13, pp. 3595-3599, 2004.

[11] K. C. Rupert, J. R. Henry, J. H. Dodd et al., "Imidazopyrimidines, potent inhibitors of p38 MAP kinase," Bioorganic and Medicinal Chemistry Letters, vol. 13, no. 3, pp. 347-350, 2003.

[12] J. T. Palmer, C. Bryant, D.-X. Wang et al., "Design and synthesis of tri-ring P3 benzamide-containing aminonitriles as potent, selective, orally effective inhibitors of cathepsin K," Journal of Medicinal Chemistry, vol. 48, no. 24, pp. 7520-7534, 2005.

[13] P. Tiew, J. R. Ioset, U. Kokpol, W. Chavasiri, and K. Hostettman, "Antifungal, antioxidant and larvicidal activities of compounds isolated from the heartwood of Mansonia gagei," Phytotherapy Research, vol. 17, no. 2, pp. 190-193, 2003.

[14] D. C. B. Da Silva-Alves, J. V. Dos Anjos, N. N. M. Cavalcante, G. K. N. Santos, D. M. D. A. F. Navarro, and R. M. Srivastava, "Larvicidal isoxazoles: synthesis and their effective susceptibility towards Aedes aegypti larvae," Bioorganic and Medicinal Chemistry, vol. 21, no. 4, pp. 940-947, 2013. 

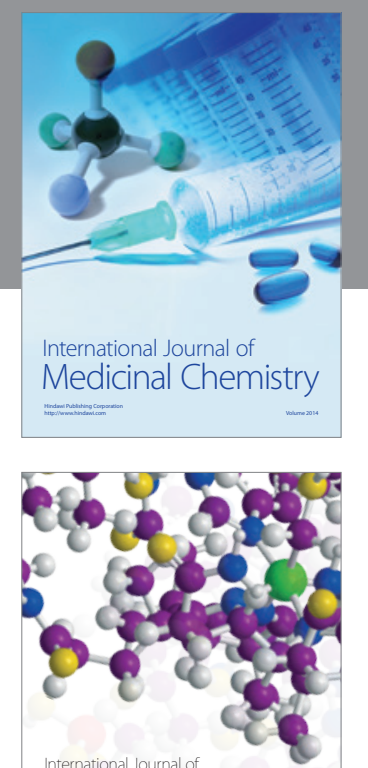

\section{Carbohydrate} Chemistry

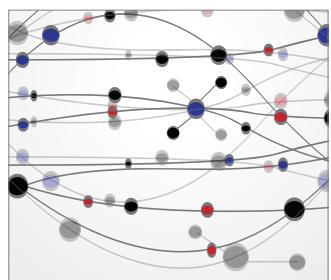

The Scientific World Journal
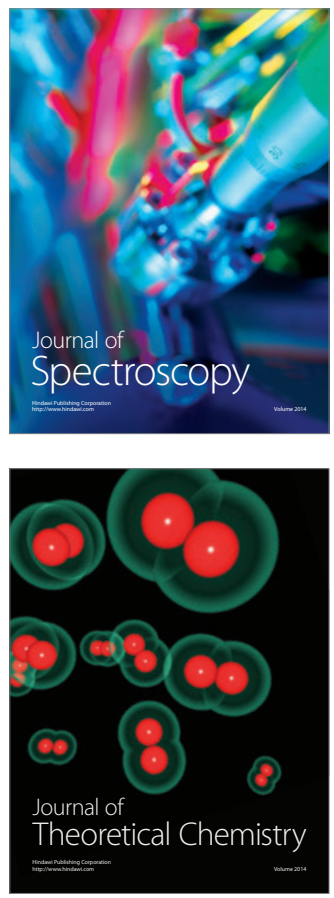
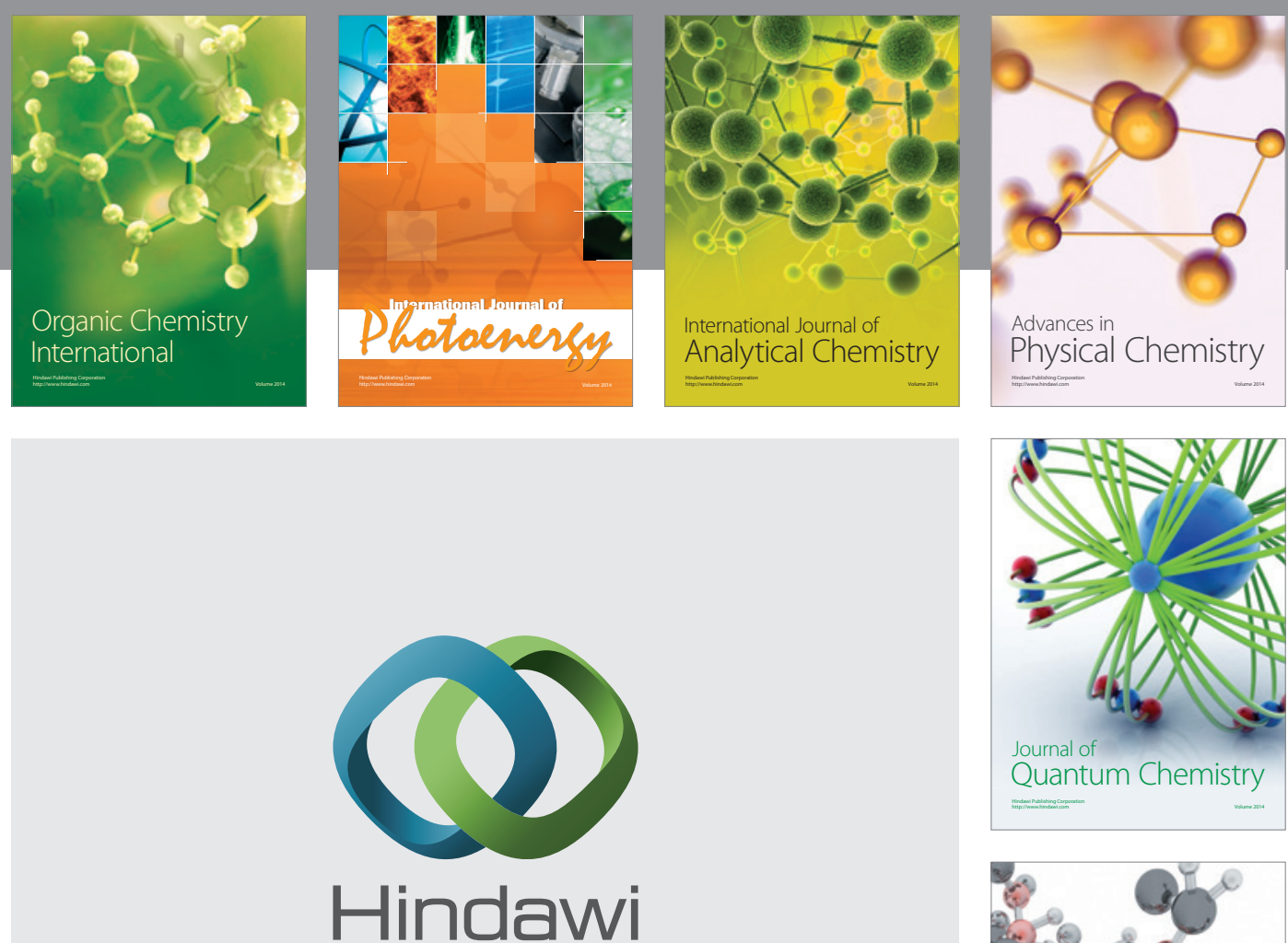

Submit your manuscripts at

http://www.hindawi.com

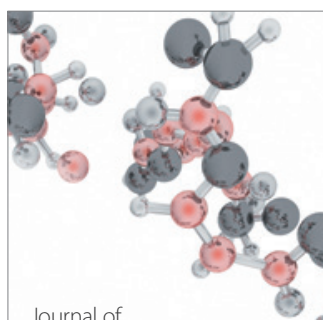

Analytical Methods

in Chemistry

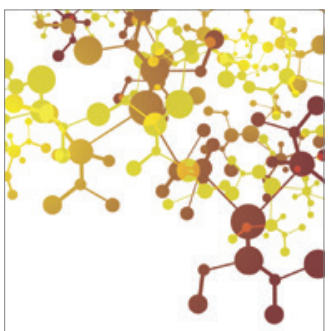

Journal of

Applied Chemistry

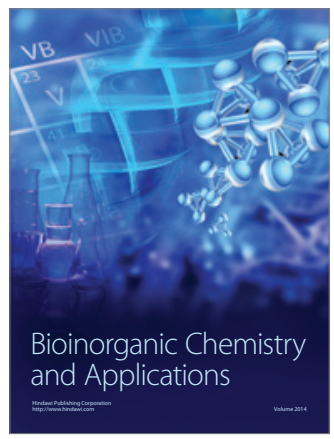

Inorganic Chemistry
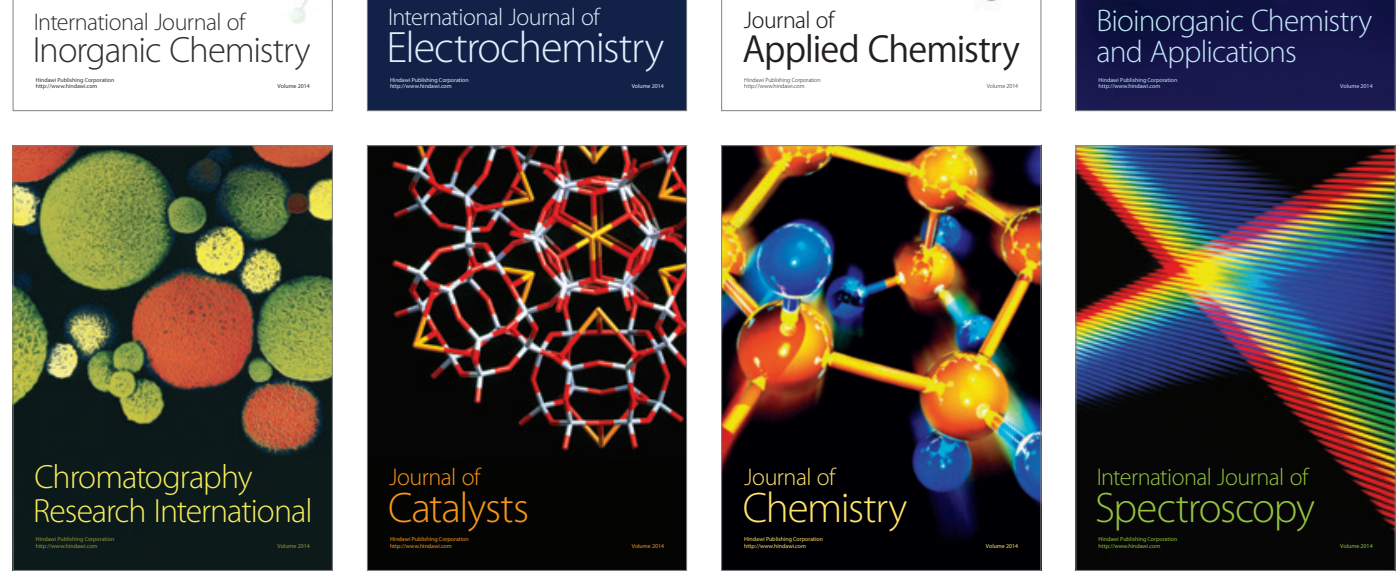